\title{
Optimal trap shape for Bose gas with attractive interactions
}

\author{
V.I. Yukalov ${ }^{1,2}$ and E.P. Yukalova ${ }^{1,3}$ \\ ${ }^{1}$ Institut für Theoretische Physik, \\ Freie Universität Berlin, Arnimallee 14, D-14195 Berlin, Germany \\ ${ }^{2}$ Bogolubov Laboratory of Theoretical Physics, \\ Joint Institute for Nuclear Research, Dubna 141980, Russia \\ ${ }^{3}$ Department of Computational Physics, Laboratory of Information Technologies, \\ Joint Institute for Nuclear Research, Dubna 141980, Russia
}

\begin{abstract}
Dilute Bose gas with attractive interactions is considered at zero temperature, when practically all atoms are in Bose-Einstein condensate. The problem is addressed aiming at answering the question: What is the optimal trap shape allowing for the condensation of the maximal number of atoms with negative scattering lengths? Simple and accurate analytical formulas are derived allowing for an easy analysis of the optimal trap shapes. These analytical formulas are the main result of the paper.
\end{abstract}

03.75.Hh, 03.65.Ge, 32.80.Pj, 11.10.Lm

Typeset using REVTEX 


\section{INTRODUCTION}

Physics of dilute Bose gases is a topic of high experimental and theoretical interest [1-5]. One of main parameters, characterizing the properties of these systems, is the intensity of atomic interactions defined by the value of the scattering length. The latter, by means of the Feshbach resonance techniques, can be varied in a wide range, including the change from positive to negative scattering lengths (see review articles [6,7] and references therein). Inverting the sign of the scattering length from positive to negative means the change of effective atomic interactions from repulsive to attractive.

The properties of Bose systems with repulsive and attractive interactions are principally different. Thus, the homogeneous Bose gas with attractive interactions is known to be unstable for any interaction intensity [8-10]. This happens because, under attractive interactions, the sound velocity of the homogeneous Bose gas becomes imaginary and, respectively, the isothermal compressibility negative and the Bogolubov spectrum complex [8-10].

The situation is different for trapped atoms, for which attractive atomic interactions can be stabilized by the zero-point kinetic energy due to the trapping potential, provided the number of atoms does not exceed a critical value $N_{c}$. Several experiments demonstrated the existence of the Bose-Einstein condensate of trapped atoms with negative scattering lengths. Thus, Bose-condensed ${ }^{7} \mathrm{Li}$ with a negative scattering length was trapped and studied in experiments [11-13]. Bose condensates of ${ }^{85} \mathrm{Rb}$, with negative scattering lengths, were obtained by varying the latter from positive to negative by means of the Feshbach resonance techniques $[14,15]$.

We may note that the Feshbach resonance techniques were also used for studying the instability in the dynamics of Bose-condensed gases, which developed owing to an abrupt change between the repulsive weak-coupling to strong-coupling regimes. For instance, in the experiment [16], a sample of ${ }^{85} \mathrm{Rb}$ atoms was evaporatively cooled and condensed into a state of atoms with a positive but a very small scattering length of about $80 a_{B}$, where $a_{B}$ is the Bohr radius. Then, by means of a fast variation of the magnetic field close to the Feshbach resonance, the scattering length was rapidly increased to $1900 a_{B}$. The dynamic instability, observed in this rapid increase of the positive scattering length has a very different physical origin [16] and will not be discussed here. In the present paper, we shall consider only equilibrium Bose-condensed gases with attractive interactions, hence, with negative scattering lengths.

Defining the critical number of Bose-condensed atoms, $\mathrm{N}_{c}$, which could be loaded into a trap, requires to accomplish heavy numerical calculations, since there are no small parameters that could facilitate the calculational procedure. The critical number $\mathrm{N}_{c}$ was calculated by different numerical methods for spherically symmetric traps [17-24] and for anysotropic traps [25-27]. The general understanding for achieving the better collapse-avoiding properties is as follows. Attractive atomic interactions are stabilized by the zero-point kinetic energy due to the trapping potential. In order to minimize the effect of attractive interactions for a given number of atoms, it is useful to reduce the condensate density, i.e., to use traps with lower confinement. The zero-point energy, corresponding to the weakest confining trap direction, is the one relevant for determining the stability limits. When the radial trap frequency was held fixed, a cigar-shaped trap had a higher critical number mostly because doing so lowered the condensate density from what it would have been if the trap 
was spherical. Eventually, the lowered confinement in the weaker direction resulted in too little zero-point energy to stabilize the atoms. Thus, there should be an optimal critical number as one of the trapping frequencies would be relaxed. If the optimal trap shape were considered under a fixed condensate density, then the spherical shape would be optimum.

Although the overall physical picture, resulting from numerical calculations [17-27], is understandable, there are no simple analytical relations allowing for a not too complicated investigation of the attractive Bose-Einstein condensate stability under the given parameters of an anisotropic trap. It is the aim of the present paper to advance a novel theoretical approach for the problem, using which we derive approximate analytical formulas connecting the critical number of atoms $N_{c}$ with the trap frequencies. Though the derivation requires the usage of some elaborated mathematics, the final expressions are quite simple. Being simple, our formulas are at the same time rather accurate. Comparing them with the known numerical calculations [17-27], we find a good agreement, with a deviation within the range of the order $10 \%$. No such analytical formulas have been obtained earlier. The derived analytical expressions can serve as a convenient tool for understanding and estimating the basic relations between the trap shape and the maximal number of trapped atoms with negative scattering lengths.

Throughout the paper the system of units is employed, where the Planck constant, $\hbar=1$, and the Boltzmann constant, $k_{B}=1$, are set to unity.

\section{CONDENSATE WAVE FUNCTION}

We consider a dilute weakly interacting Bose gas, corresponding to the inequality $\rho\left|a_{s}\right|^{3} \ll 1$, in which $\rho$ is the average atomic density and $a_{s}$ is the $s$-wave scattering length. For repulsive interactions, $a_{s}>0$, while for attractive interactions, $a_{s}<0$. The dilute Bose gas in the low-temperature limit becomes almost completely condensed, forming BoseEinstein condensate with the number of atoms $N_{0}$ being approximately equal to the total number of atoms $N$. The condensate wave function, as is known [1-5], satisfies the GrossPitaevskii equation

$$
\left[-\frac{\nabla^{2}}{2 m}+U(\mathbf{r})+\Phi_{0}|\eta(\mathbf{r})|^{2}\right] \eta(\mathbf{r})=\varepsilon \eta(\mathbf{r}),
$$

where $m$ is atomic mass, $U(\mathbf{r})$ is a trapping potential, and the interaction strength is

$$
\Phi_{0}=4 \pi \frac{a_{s}}{m} .
$$

Equation (1) has the form of the stationary nonlinear Schrödinger equation representing the eigenvalue problem, with the eigenfunction $\eta(\mathbf{r})$ and eigenvalue $\varepsilon$. The confining potential is usually described by the harmonic potential

$$
U(\mathbf{r})=\frac{m}{2} \sum_{\alpha} \omega_{\alpha}^{2} r_{\alpha}^{2}
$$

whose effective frequencies $\omega_{\alpha}$ define the anisotropy of the trap. The condensate wave function is normalized to the number of condensed atoms, 


$$
\int|\eta(\mathbf{r})|^{2} d \mathbf{r}=N_{0}
$$

Under the considered conditions, this number is close to the total number of atoms, $N_{0} \approx N$.

The standard traps usually have the geometry of a cylinder or are almost cylindrical, which we assume in what follows. Then there are two characteristic trap frequencies, the transverse, or radial, frequency

$$
\omega_{\perp} \equiv \omega_{x}=\omega_{y}
$$

and the longitudinal, or axial, frequency $\omega_{z}$. The anisotropy parameter

$$
\lambda \equiv \frac{\omega_{z}}{\omega_{\perp}}
$$

defines the actual trap shape. When $\lambda<1$, the trap is called cigar-shaped, or pencil-shaped. For $\lambda=1$, the trap is spherical. And when $\lambda>1$, one terms the trap as disk-shaped, or pancake-shaped. Related to the characteristic frequencies $\omega_{\perp}$ and $\omega_{z}$, there are two oscillator lengths

$$
l_{\perp} \equiv \frac{1}{\sqrt{m \omega_{\perp}}}, \quad l_{z} \equiv \frac{1}{\sqrt{m \omega_{z}}}
$$

One more typical length is defined through the geometric-average frequency $\omega_{0}$,

$$
l_{0} \equiv \frac{1}{\sqrt{m \omega_{0}}}, \quad \omega_{0} \equiv\left(\omega_{\perp}^{2} \omega_{z}\right)^{1 / 3} .
$$

From Eqs. (5) to (8), it is straightforward to notice the relations between the characteristic frequencies,

$$
\omega_{0}=\lambda^{1 / 3} \omega_{\perp}=\frac{\omega_{z}}{\lambda^{2 / 3}},
$$

and between the oscillator lengths,

$$
l_{0}=\frac{l_{\perp}}{\lambda^{1 / 6}}=\lambda^{1 / 3} l_{z}
$$

Employing the cylindrical coordinates $\mathbf{r}=\left\{r_{\perp}, \varphi, r_{z}\right\}$, where $r_{\perp}=\sqrt{r_{x}^{2}+r_{y}^{2}}$, it is convenient to introduce the dimensionless variables

$$
r \equiv \frac{r_{\perp}}{l_{\perp}}, \quad z \equiv \frac{r_{z}}{l_{\perp}} .
$$

The equality

$$
\eta(\mathbf{r}) \equiv \sqrt{\frac{N_{0}}{l_{\perp}^{3}}} \psi(r, \varphi, z)
$$

defines the dimensionless wave function $\psi(r, \varphi, z)$ of the radial variable $r \in[0, \infty)$, angle variable $\varphi \in[0,2 \pi)$, and of the longitudinal variable $z \in(-\infty,+\infty)$. The normalization condition (4) transforms to 


$$
\int|\psi(r, \varphi, z)|^{2} r d r d \varphi d z=1
$$

With the notation for the dimensionless energy

$$
E \equiv \frac{\varepsilon}{\omega_{\perp}}
$$

the Gross-Pitaevskii equation (1) takes the form of the eigenvalue problem

$$
\hat{H}_{N L S} \psi=E \psi,
$$

in which the nonlinear Schrödinger Hamiltonian is

$$
\hat{H}_{N L S} \equiv-\frac{\nabla^{2}}{2 m}+\frac{1}{2}\left(r^{2}+\lambda^{2} z^{2}\right)+g|\psi|^{2} .
$$

Here

$$
\nabla^{2}=\frac{\partial^{2}}{\partial r^{2}}+\frac{1}{r} \frac{\partial}{\partial r}+\frac{1}{r^{2}} \frac{\partial^{2}}{\partial \varphi^{2}}+\frac{\partial^{2}}{\partial z^{2}}
$$

and the notation for the effective coupling parameter

$$
g \equiv 4 \pi \frac{a_{s}}{l_{\perp}} N_{0}
$$

is introduced.

Equation (15), with the Hamiltonian (16), clearly shows that the energy $E$ is a function of $g$ and $\lambda$. Real-valued solutions for the eigenvalue $E=E(g, \lambda)$ exist not for all parameters $g$ and $\lambda$. For some values of these parameters, the eigenvalue $E$ can become complex. The appearance of the imaginary part in the energy $E$, means that the state with this energy level becomes unstable, having a finite lifetime that can be estimated as

$$
\tau \equiv \frac{1}{|\operatorname{Im} E|}
$$

The boundary on the plane $g-\lambda$, where real-valued solutions for $E$ disappear, defines the critical line $g_{c}(\lambda)$, which, through the relation

$$
g_{c}(\lambda)=4 \pi \frac{a_{s}}{l_{\perp}} N_{c}
$$

gives the critical number of atoms $N_{c}$. For the number of atoms $N_{0}<N_{c}$, the eigenproblem (15) possesses well-defined solutions, with real-valued energies. But, if $N_{0}$ exceeds $N_{c}$, the energy becomes complex, which means that the system is unstable and desintegrates. This critical number follows from Eq. (18) yielding

$$
N_{c}=\frac{g_{c}(\lambda)}{4 \pi}\left(\frac{l_{\perp}}{a_{s}}\right)=\frac{g_{c}(\lambda)}{4 \pi} \lambda^{1 / 2}\left(\frac{l_{z}}{a_{s}}\right) .
$$

Or, according to Eq. (10), one has

$$
N_{c}=\frac{g_{c}(\lambda)}{4 \pi} \lambda^{1 / 6}\left(\frac{l_{0}}{a_{s}}\right)
$$

To find the critical line $g_{c}(\lambda)$, it is necessary to solve Eq. (15) for varied parameters $g$ and $\lambda$. Directly solving this nonlinear equation, with an external potential, requires to invoke cumbersome numerical calculations. It would certainly be desirable to obtain an explicit analytical expression for the critical line $g_{c}(\lambda)$, which would permit us to accomplish a straightforward investigation of Eqs. (19) and (20). 


\section{OPTIMIZED PERTURBATION THEORY}

The behaviour of the energy $E$, as a function of $g$ and $\lambda$, can be found by using the optimized perturbation theory. This theory, advanced in Ref. [28], is based on introducing into the calculational procedure, such as perturbation theory or iterative algorithm, control functions, which govern the convergence of the calculational scheme. Control functions can be incorporated either into the initial approximation or into the resulting series by changing the variables. Then, to guarantee the convergence of the sequence of approximations, the control functions are defined by means of an optimization procedure. This results in the sequence of optimized approximants. The specific features of the optimized perturbation theory is its high accuracy and the possibility of its usage for the problems with no small parameters. This theory is nowadays widely employed for various problems (see the reviewtype papers $[29,30]$ and references therein). In particular, it has been successfully applied for calculating the interaction-induced shift of the condensation temperature of dilute Bose gas [31-36], yielding very accurate results, well agreeing with numerical Monte Carlo simulations $[37-41]$.

To solve Eq. (15), we may start with the zero-order Hamiltonian

$$
\hat{H}_{0}=-\frac{\nabla^{2}}{2 m}+\frac{1}{2}\left(u^{2} r^{2}+\lambda^{2} v^{2} z^{2}\right)
$$

in which $u$ and $v$ are control functions. The corresponding zero-order energy is

$$
E_{0}=(2 n+|m|+1) u+\left(k+\frac{1}{2}\right) \lambda v
$$

in which $n=0,1,2, \ldots$ is the radial quantum number, $m=0, \pm 1, \pm 2, \ldots$ is the azimuthal quantum number, and $k=0,1,2, \ldots$ is the axial quantum number. Employing the RayleighSchrödinger perturbation theory, we get a sequence of the approximants

$$
E_{j}=E_{j}(g, \lambda, u, v) .
$$

For brevity, we do not include explicitly the dependence on the quantum numbers. The control functions are defined in each order through optimization conditions, e.g., through the variation

$$
\delta E_{j}=\frac{\partial E_{j}}{\partial u} \delta u+\frac{\partial E_{j}}{\partial v} \delta v=0
$$

which gives the functions

$$
u_{j}=u_{j}(g, \lambda), \quad v_{j}=v_{j}(g, \lambda) .
$$

Substituting these back into Eq. (23), we obtain the optimized approximants

$$
\tilde{E}_{j} \equiv E_{j}\left(g, \lambda, u_{j}, v_{j}\right)
$$

with the control functions (25).

To simplify the following expressions, it is useful to introduce an effective coupling 


$$
x \equiv 2 g \sqrt{\lambda} I
$$

where the integral

$$
I \equiv \frac{\left(|\psi|^{2},|\psi|^{2}\right)}{u \sqrt{\lambda v}}
$$

is defined through the matrix element of the wave functions $\psi$ corresponding to the Hamiltonian (21). Explicitly, the wave functions are

$$
\begin{gathered}
\psi(r, \varphi, z)=\left[\frac{2 n ! u^{|m|+1}}{(n+|m|) !}\right]^{1 / 2} r^{|m|} \exp \left(-\frac{u}{2} r^{2}\right) \times \\
\times L_{n}^{|m|}\left(u r^{2}\right) \frac{e^{i m \varphi}}{\sqrt{2 \pi}} \frac{1}{\sqrt{2^{k} k !}}\left(\frac{\lambda v}{\pi}\right)^{1 / 4} \exp \left(-\frac{\lambda}{2} v z^{2}\right) H_{k}(\sqrt{\lambda v} z),
\end{gathered}
$$

where $L_{n}^{m}(\cdot)$ is an associated Laguerre polynomial and $H_{k}(\cdot)$ is a Hermite polynomial. Then the integral (28) takes the form

$$
I=\frac{2}{\pi^{2}}\left[\frac{n !}{(n+|m|) ! 2^{k} k !}\right]^{2} \int_{0}^{\infty} x^{2|m|} e^{-2 x}\left[L_{n}^{|m|}(x)\right]^{4} d x \int_{0}^{\infty} e^{-2 t^{2}} H_{k}^{4}(t) d t,
$$

which is a number not depending on the control functions $u$ and $v$. In the first order, we have the energy

$$
E_{1}=\frac{p}{2}\left(u+\frac{1}{u}\right)+\frac{q}{4}\left(v+\frac{1}{v}\right)+\frac{1}{2} u \sqrt{v} x
$$

in which the notation

$$
p \equiv 2 n+|m|+1, \quad q \equiv(2 k+1) \lambda
$$

is used. The variation of Eq. (29) defines the control functions by the optimization equations

$$
1-\frac{1}{u^{2}}+\frac{\sqrt{v}}{p} x=0, \quad 1-\frac{1}{v^{2}}+\frac{u x}{q \sqrt{v}}=0 .
$$

These equations are to be supplemented by the boundary conditions

$$
\lim _{x \rightarrow 0} u=\lim _{x \rightarrow 0} v=1,
$$

requiring that, when atomic interactions are switched off, the solutions be recovered corresponding to a harmonic oscillator. By their meaning, the control functions play the role of effective frequencies, because of which they have to be positive. From Eqs. (31) it follows that the range of definition for the solutions $u$ and $v$ depends on the sign of atomic interactions. Thus, for repulsive interactions, when $g>0$ and $x>0$, these solutions are to be in the interval

$$
0<u<1, \quad 0<v<1 \quad(x>0) .
$$


But for attractive interactions, with $g<0$ and $x<0$, one has

$$
u>1, \quad v>1 \quad(x<0) .
$$

Equation (29), together with the optimization conditions (31), gives the optimized approximant $\tilde{E}=\tilde{E}_{1}$, which we shall analyze in what follows. This optimized approximant can be written in the form

$$
\tilde{E}=\frac{p}{u}+\frac{q}{4}\left(v+\frac{1}{v}\right)
$$

in which $u$ and $v$ are the solutions to Eqs. (31).

The set of Eqs. (31) and (35) defines the control functions $u$ and $v$ and the optimized approximant $\tilde{E}$ as functions of the effective coupling (27) and quantum numbers (30). Explicit dependence on these parameters can be obtained in the limits of weak and strong coupling, as is demonstrated in the Appendix A.

Of special interest is the behaviour of the ground-state level, which, in the case of attractive interactions, becomes unstable before the higher excited levels. For the ground state, one has $n=m=k=0$, hence $p=1$ and $q=\lambda$. The integral (28) is $I=(2 \pi)^{-3 / 2}$. The optimization equations (31) reduce to

$$
1-\frac{1}{u^{2}}+\sqrt{v} x=0, \quad 1-\frac{1}{v^{2}}+\frac{u x}{\lambda \sqrt{v}}=0,
$$

where the effective coupling (27) is

$$
x=\frac{2 g \sqrt{\lambda}}{(2 \pi)^{3 / 2}} .
$$

The optimized approximant (35) becomes

$$
\tilde{E}=\frac{1}{u}+\frac{\lambda}{4}\left(v+\frac{1}{v}\right)
$$

Expression (38) represents the ground-state energy with a very good accuracy for the whole range of the interaction strength, from zero to arbitrary large values. Thus, for weak coupling, Eq. (38) gives an asymptotically exact value

$$
\tilde{E} \simeq 1+\frac{\lambda}{2}+\frac{g \sqrt{\lambda}}{(2 \pi)^{3 / 2}} \quad(g \rightarrow \pm 0) .
$$

The accuracy slightly diminishes for increasing interactions. However, even in the limit of infinitely strong repulsive interactions, Eq. (38) yields the limit

$$
\tilde{E} \simeq 0.547538(g \lambda)^{2 / 5} \quad(g \rightarrow \infty),
$$

which is only $2 \%$ different from the Thomas-Fermi limit that is known to be asymptotically exact for $g \rightarrow \infty$. For large attractive interactions, when $g<0$, there is no simple asymptotic form for $g \rightarrow-\infty$, since the system becomes unstable at a finite critical value $g_{c}(\lambda)$. The consideration of attractive interactions is more complicated and, to find explicitly the critical line $g_{c}(\lambda)$, we need to involve some more techniques. 


\section{ATTRACTIVE ATOMIC INTERACTIONS}

Now the consideration of the previous section will be continued with the specification for attractive interactions, when $g<0$ and $x<0$. First of all, let us note that real-valued solutions for the ground-state energy (38), with the control functions from Eqs. (36), exist only for $x>x_{c}$, such that

$$
\left|x_{c}\right|<1 \quad(0 \leq \lambda<\infty) .
$$

The proof of this inequality is as follows. Equations (36) can be rearranged to the form

$$
\frac{\lambda^{2}}{x^{2}}(1-|x| \sqrt{v})=\frac{v^{3}}{\left(v^{2}-1\right)^{2}} .
$$

As far as the right-hand side of Eq. (42) is positive, it should be that

$$
1-|x| \sqrt{v} \geq 0 .
$$

From here, $|x| \leq 1 / \sqrt{v}$. Since, according to Eq. (34), $v>1$, if $x<0$, then $|x|<1$. The latter results in Eq. (41).

Because the critical value is smaller than one, $\left|x_{c}\right|<1$, for any anisotropy parameter $\lambda \geq 0$, it is reasonable to study, first, the situation at asymptotically small $|x| \leq\left|x_{c}\right|<1$. Making the replacement $x=-|x|$, we may analyze the behaviour at small $|x|$ for the control functions as well as for the energy. Excluding $v$ in the optimization conditions (36), we get the equation

$$
x^{2}\left(u^{2}-1\right)^{3} u^{3}-\lambda\left(u^{2}-1\right)^{4}+\lambda x^{4} u^{8}=0
$$

for the control function $u$. Vice versa, excluding $u$, we have Eq. (42) for the control function $v$. Energy (38) can be represented in terms of one of these functions, for instance,

$$
\tilde{E}=\frac{1}{u}+\frac{\lambda}{4}\left[\frac{\left(u^{2}-1\right)^{2}}{x^{2} u^{4}}+\frac{x^{2} u^{4}}{\left(u^{2}-1\right)^{2}}\right] .
$$

Using Eqs. (42),(43),(38) or (44), we can find the expansions for all functions in powers of $|x|$. The expansions for the control functions are

$$
u \simeq \sum_{k=0}^{j} u_{k}|x|^{k}
$$

and

$$
v \simeq \sum_{k=0}^{j} v_{k}|x|^{k}
$$

The expansion for the energy is

$$
\tilde{E} \simeq \sum_{k=0}^{j} c_{k}|x|^{k}
$$


In Eqs. (45) to (47), $j=0,1,2, \ldots$. The first several coefficients for these expansions are given in the Appendix B.

Expansions (45) to (47) have sense for asymptotically small $|x| \ll 1$. The value $x_{c}(\lambda)$, should be smaller than one, in agreement with Eq. (41). However, $\left|x_{c}\right|$ is not necessarily asymptotically small. This can be illustrated by the spherically symmetric case, when $\lambda=1$. Then $u=v$, and Eqs. (31) reduce to just one equation

$$
x u^{5 / 2}+u^{2}-1=0 \quad(\lambda=1) .
$$

The latter equation possesses real solutions only for $x>x_{c}$, such that

$$
x_{c}=-\frac{4}{5^{5 / 4}}=-0.534992,
$$

which is related to $g_{c}=-4.212958$. Note that the critical value (49) follows exactly from Eq. (48).

In this way, we need more general expressions for the sought functions, but not merely their asymptotic expansions (45) to (47). This can be achieved by resorting to a method of an effective summation of power series. A very accurate method of summation, whose mathematical foundation is based on the self-similar approximation theory [42-46], is the method of self-similar factor approximants [47-49], which was shown to be more general and accurate than the method of Padé approximants, the latter being just a particular case on the class of rational functions.

The self-similar factor approximant of the $j$-th order for a power series, say for expansion (45), has the form

$$
u_{j}^{*}=u_{0} \prod_{i=1}^{N_{j}}\left(1+A_{i}|x|\right)^{n_{i}},
$$

in which $N_{j}=j / 2$, when $j$ is even, and $N_{j}=(j+1) / 2$, with $A_{1}$ set to one, if $j$ is odd. The coefficients $A_{i}$ and the powers $n_{i}$ are defined by the accuracy-through-order procedure, that is, by expanding the approximant (50) in power series with respect to $|x|$ and equating in that expansion the same-order terms with those of series (45). In the same way the approximants $v_{j}^{*}$ for another control function are constructed. And, similarly, for the energy we obtain

$$
E_{j}^{*}=c_{0} \prod_{i=1}^{N_{j}}\left(1+C_{i}|x|\right)^{m_{i}} .
$$

The factor approximants (50) and (51) extrapolate the validity of the asymptotic series (45) and (47) to the whole region of finite $|x|$. As has been shown [47-49], such an extrapolation works very well for the whole region of the variable, from zero to infinity, being of especially good accuracy for the region between zero and the values of the variable of order one. It is exactly the latter region $0 \leq|x| \leq\left|x_{c}\right|<1$, which we here deal with.

Having in hands the analytical expressions for the sought functions $u_{j}^{*}, v_{j}^{*}$, and $E_{j}^{*}$, we are already close to our aim of finding an analytical form of the critical line $x_{c}(\lambda)$ and, respectively, $g_{c}(\lambda)$. For this purpose, it is sufficient to investigate the behaviour of the 
functions (50) and (51), with varying $|x|$ from zero to the point $\left|x_{c}\right|$, where real positive solutions stop existing. The critical line $x_{c}(\lambda)$ defines, according to relation (37), the critical line for the coupling

$$
g_{c}(\lambda)=\frac{(2 \pi)^{3 / 2}}{2 \sqrt{\lambda}} x_{c}(\lambda)
$$

The latter, by definition (18), gives the critical number of atoms $N_{c}$ as a function of the anisotropy parameter $\lambda$.

However, as relations (19) and (20) show, the critical number of atoms is not expressed solely through the anisotropy parameter $\lambda$, but also involves one of the characteristic lengths (7) or (8). So, $N_{c}$ is a function of two parameters, assuming that the scattering length is fixed. For instance, if we choose the transverse length $l_{\perp}$ as one of the parameters and define

$$
\alpha_{\perp}(\lambda) \equiv \frac{1}{4 \pi}\left|g_{c}(\lambda)\right|
$$

then the critical number of atoms is

$$
N_{c}=\alpha_{\perp}(\lambda)\left|\frac{l_{\perp}}{a_{s}}\right| .
$$

But if the axial length $l_{z}$ is chosen, then, with the notation

$$
a_{z}(\lambda) \equiv \frac{\sqrt{\lambda}}{4 \pi}\left|g_{c}(\lambda)\right|,
$$

we have the critical number

$$
N_{c}=\alpha_{z}(\lambda)\left|\frac{l_{z}}{a_{s}}\right| .
$$

Finally, fixing the length $l_{0}$, and using the notation

$$
\alpha_{0}(\lambda) \equiv \frac{\lambda^{1 / 6}}{4 \pi}\left|g_{c}(\lambda)\right|
$$

we get the critical number

$$
N_{c}=\alpha_{0}(\lambda)\left|\frac{l_{0}}{a_{s}}\right| .
$$

These formulas demonstrate that $N_{c}$ depends on two parameters. Therefore, maximazing one of the critical couplings (53), (55), or (57) for finding a maximal $N_{c}$ implies a conditional variation under one of the characteristic lengths being fixed.

To find the conditional maxima of $N_{c}$, we need to define the critical line $x_{c}(\lambda)$, where positive solutions for functions (50) or (51) stop existing. To estimate the accuracy of the obtained critical values $x_{c}$, we constructed the factor approximants (50) and (51) up to the 17-th order and compared the values of $x_{c}$ for $\lambda=1$ with the exact value (49). The comparison shows good numerical convergence of the factor approximants, the sequence $\left\{u_{j}^{*}\right\}$ 
converging a little faster than $\left\{E_{j}^{*}\right\}$, because of which the values of $x_{c}$ obtained from the former sequence are more accurate. We shall take this into account in what follows, obtaining $x_{c}$ from the most accurate sequence. The error of $x_{c}$, found from the factor approximants up to the 5 -th order, is of the order of $10 \%$. After the fifth-order approximant, the error quickly diminishes to about $1 \%$. We compared in detail the behaviour of $x_{c}(\lambda)$ and, respectively, of $g_{c}(\lambda)$, found from the factor approximants up to the fifth order, in the whole range of the anisotropy parameter $\lambda$. This behaviour of $g_{c}(\lambda)$ translates into the properties of the related critical couplings (53), (55), and (57). Thus, for the transverse coupling (53), the limiting behaviour at very small or very large anisotropy parameters is

$$
\begin{gathered}
\alpha_{\perp}(\lambda) \simeq 1.253 \lambda^{1 / 2} \quad(\lambda \rightarrow 0), \\
\alpha_{\perp}(\lambda) \simeq 0.627 \lambda^{-1 / 2} \quad(\lambda \rightarrow \infty) .
\end{gathered}
$$

For the axial coupling (55), we find the limits

$$
\begin{gathered}
\alpha_{z}(\lambda) \simeq 1.253 \lambda \quad(\lambda \rightarrow 0), \\
\alpha_{z}(\lambda) \simeq 0.627 \quad(\lambda \rightarrow \infty) .
\end{gathered}
$$

And for the critical coupling (57), we get

$$
\begin{gathered}
\alpha_{0}(\lambda) \simeq 1.253 \lambda^{2 / 3} \quad(\lambda \rightarrow 0), \\
\alpha_{0}(\lambda) \simeq 0.627 \lambda^{-1 / 3} \quad(\lambda \rightarrow \infty) .
\end{gathered}
$$

Choosing between the critical lines $x_{c}(\lambda)$, obtained from the factor approximants of different orders, we keep in mind our main aim of deriving sufficiently simple analytical expressions that would be convenient to study. The accuracy of the second- and third-order factor approximants are very close to each other, with the errors of the order of $10 \%$. The accuracy of the fourth-order approximant is slightly better, but the expression for $x_{c}(\lambda)$ is much more cumbersome. Therefore, we limit ourselves by the second-order approximant, which gives the critical line

$$
x_{c}(\lambda)=-\frac{2 \lambda}{1+2 \lambda}
$$

Respectively, the critical line for the effective coupling (52) is

$$
g_{c}(\lambda)=-(2 \pi)^{3 / 2} \frac{\sqrt{\lambda}}{1+2 \lambda} .
$$

The higher-order versions of the critical lines, with the comparison of their properties are given in the Appendix C. From Eq. (53), we obtain the radial critical coupling,

$$
\alpha_{\perp}=\sqrt{\frac{\pi}{2}}\left(\frac{\sqrt{\lambda}}{1+2 \lambda}\right),
$$

the axial critical coupling (55), 


$$
\alpha_{z}=\sqrt{\frac{\pi}{2}}\left(\frac{\lambda}{1+2 \lambda}\right)
$$

and the average coupling (57),

$$
\alpha_{0}=\sqrt{\frac{\pi}{2}}\left(\frac{\lambda^{2 / 3}}{1+2 \lambda}\right) .
$$

These simple formulas allow us to accomplish a straightforward search for an optimal trap shape. As is explained above, the result depends on the constraint under which the maximal critical number $N_{c}$ is defined. Thus, fixing the radial trap frequency $\omega_{\perp}$, hence, the radial length $l_{\perp}$, and varying the anisotropy parameter $\lambda$, one has to maximize the radial critical coupling (64), which gives

$$
\max _{\lambda} \alpha_{\perp}(\lambda)=0.443 \quad(\lambda=0.5)
$$

This tells us that, under the given conditions, the cigar-shaped trap, with $\lambda=0.5$, will be able to confine the maximal number of atoms (54).

If one chooses to fix the axial trap frequency, $\omega_{z}$, hence the axial length $l_{z}$, then one should look for the maximum of the axial critical coupling (65), which yields

$$
\max _{\lambda} \alpha_{z}(\lambda)=0.627 \quad(\lambda \rightarrow \infty) .
$$

This means that, under the chosen conditions, the disk-shaped trap can contain the maximal number of atoms (56).

And, if one prefers to fix the average trap frequency $\omega_{0}$, hence the length $l_{0}$, then the average critical coupling (66) is to be maximized, which leads to

$$
\max _{\lambda} \alpha_{0}(\lambda)=0.418 \quad(\lambda=1) .
$$

Under these conditions, the spherical trap is preferable, if one wishes to confine the maximal number of atoms (58).

These conclusions are in agreement with the general physical picture discussed in the Introduction. Let us emphasize that if the optimal trap shape is considered under a fixed condensate density at the trap center

$$
\rho(0) \sim N\left(\frac{m \omega_{0}}{\pi}\right)^{3 / 2}
$$

that is, under a fixed $\omega_{0}$, then the spherically symmetric trap is optimal, in agreement with the above analysis. Note also that fixing the density $\rho(0)$, or frequency $\omega_{0}$, is equivalent to fixing the condensation temperature

$$
T_{c} \sim \omega_{0}\left[\frac{N}{\zeta(3)}\right]^{1 / 3},
$$

where $\zeta(3)$ is the Rieman zeta function. 
For the purpose of estimating the critical number of atoms with attractive interactions for a given trap with the fixed trap frequencies, that is, with the given characteristic lengths, it is convenient to express the critical numbers (54), (56), and (58) directly through the trap parameters. Then, from any of these expressions, we obtain

$$
N_{c}=\sqrt{\frac{\pi}{2}} \frac{l_{x} l_{y} l_{z}}{\left|a_{s}\right|\left(l_{x}^{2}+l_{y}^{2}+l_{z}^{2}\right)} .
$$

It is convenient to define the dimensionless quantity

$$
N_{c} \frac{\left|a_{s}\right|}{l_{0}}=\sqrt{\frac{\pi}{2}} \frac{l_{0}^{2}}{l_{x}^{2}+l_{y}^{2}+l_{z}^{2}},
$$

where $l_{0} \equiv\left(l_{x} l_{y} l_{z}\right)^{1 / 3}$. Equation (68) can be rewritten in terms of the trap frequencies. Introducing the notation for the average inverse frequency $\omega_{i n v}$ through the equality

$$
\frac{1}{\omega_{i n v}} \equiv \frac{1}{3}\left(\frac{1}{\omega_{x}}+\frac{1}{\omega_{y}}+\frac{1}{\omega_{z}}\right),
$$

we find

$$
N_{c} \frac{\left|a_{s}\right|}{l_{0}}=\sqrt{\frac{\pi}{2}}\left(\frac{\omega_{i n v}}{3 \omega_{0}}\right) .
$$

Formulas (67) to (70) can serve as a useful tool for a fast and simple estimations of the critical number of atoms, with a negative scattering length, which could be loaded into a given trap.

\section{CONCLUSION}

We have considered a dilute gas of trapped Bose atoms at zero temperature, when practically all atoms are in the ground state of Bose-Einstein condensate. Atoms are interacting through attractive forces, corresponding to negative scattering lengths. The number of possible atoms in such a Bose-Einstein condensate is limited by a critical number $N_{c}$ depending on the trap parameters. The main result of the present paper is the derivation of simple analytical expressions for estimating this critical number $N_{c}$. The investigation of the derived formulas clearly shows that the number of atoms $N_{c}$ cannot be expressed through the sole parameter of anisotropy. Therefore, in order to answer the question "What is the optimal trap shape for keeping the maximal number of atoms with attractive interactions", it is necessary to specify under what conditions one is looking for this maximum. Thus, if the radial trap frequency $\omega_{\perp}$ is kept fixed and the anisotropy parameter $\lambda$ is varied, then the cigar-shaped trap with $\lambda=0.5$ is optimal. But if the axial trap frequency $\omega_{z}$ is fixed, then the disc-shaped trap is optimal. And when the geometric-average frequency $\omega_{0}$ is fixed, with the anisotropy parameter being varied, then the spherically symmetric trap becomes optimal. The spherical trap is also optimal, when the condensate density is kept fixed. In general, for estimating the critical number of atoms $N_{c}$, it is convenient to use formulas (67) to (70), which are represented in the form valid for arbitrary trap frequencies $\omega_{x}, \omega_{y}$, and 
$\omega_{z}$, hence, for arbitrary trap lengths $l_{x}, l_{y}$, and $l_{z}$. It is easy to check that these estimates are in good agreement with experiment.

For instance, in the experiments $[12,50]$ with ${ }^{7} \mathrm{Li}$, having the negative scattering length $a_{s}=-27.3 a_{B}=-14.5 \AA$, where $a_{B}$ is the Bohr radius, the trap with the frequencies $\omega_{x}=2 \pi \times 150.6 \mathrm{~Hz}, \omega_{y}=2 \pi \times 152.6 \mathrm{~Hz}$, and $\omega_{z}=2 \pi \times 131.5 \mathrm{~Hz}$ was used. This, for the mass $m_{\mathrm{Li}}=11.6 \times 10^{-24} \mathrm{~g}$, translates into the characteristic lengths $l_{x}=0.309 \times 10^{-3} \mathrm{~cm}$, $l_{y}=0.307 \times 10^{-3} \mathrm{~cm}$, and $l_{z}=0.331 \times 10^{-3} \mathrm{~cm}$. In these experiments [12,50] the critical number was estimated being between 600 and 1300 atoms, that is, on average, $N_{c} \approx 950$. Our formula (67), for this case, gives $N_{c}=910$.

In the experiments $[14,15]$ with ${ }^{85} \mathrm{Rb}$, the scattering length could be varied by means of the Feshbach resonance techniques. The trap had the frequencies $\omega_{x}=2 \pi \times 17.24 \mathrm{~Hz}$, $\omega_{y}=2 \pi \times 17.47 \mathrm{~Hz}$, and $\omega_{z}=2 \pi \times 6.80 \mathrm{~Hz}$, that is $\omega_{0}=2 \pi \times 12.7 \mathrm{~Hz}$. The dimensionless parameter (70) was found to be equal to $N_{c}\left|a_{s}\right| / l_{0}=0.46 \pm 0.07$. From our formula (70) for this trap, we find $N_{c}\left|a_{s}\right| / l_{0}=0.47$.

In this way, the derived formulas allow one to quickly get rather accurate estimates for the critical number of atoms. The advantage of these formulas is their simple analytical representation. Therefore, to find $N_{c}$ and to optimize the trap shape, one does not need to plunge into lengthy and complicated numerical calculations, but it is sufficient to employ the derived simple analytical formulas.

\section{Acknowledgement}

We are grateful for the financial support to the German Research Foundation. 


\section{Appendix A. Properties of Optimized Approximants}

The control functions and the optimized approximant for the dimensionless energy, discussed in Sec. III, have the following asymptotic behaviour in the weak-coupling and strongcoupling limits.

In the weak-coupling limit, when $g \rightarrow \pm 0$, the effective coupling parameter (27) is such that $x \rightarrow \pm 0$. Then from the optimization equations (31), we find

$$
\begin{aligned}
& u \simeq 1-\frac{1}{2 p} x+\frac{p+3 q}{8 p^{2} q} x^{2}-\frac{3 p^{2}+16 p q+20 q^{2}}{64 p^{3} q^{2}} x^{3}, \\
& v \simeq 1-\frac{1}{2 q} x+\frac{p+q}{4 p q^{2}} x^{2}-\frac{7 p^{2}+20 p q+12 q^{2}}{64 p^{2} q^{3}} x^{3} .
\end{aligned}
$$

These expressions satisfy the boundary conditions (32).

In the strong-coupling limit, when $g \rightarrow \infty$ and $x \rightarrow \infty$, we have the control functions

$$
\begin{gathered}
u \simeq\left(\frac{p^{3}}{q}\right)^{1 / 5} x^{-2 / 5}+\frac{q^{2}-3 p^{2}}{5\left(p q^{3}\right)^{1 / 5}} x^{-6 / 5}+\frac{3 p^{4}-p^{2} q^{2}-q^{4}}{5 p q} x^{-2}, \\
v \simeq\left(\frac{q^{2}}{p}\right)^{2 / 5} x^{-2 / 5}+\frac{2\left(p^{2}-2 q^{2}\right)}{5}\left(\frac{q}{p^{3}}\right)^{2 / 5} x^{-6 / 5}+\frac{6 q^{4}-4 p^{2} q^{2}-p^{4}}{5 p^{2}} x^{-2} .
\end{gathered}
$$

The strong-coupling limit for attractive interactions, for which $x \rightarrow-\infty$, is not defined, since the solutions to Eqs. (31) become complex.

The weak-coupling limit for the optimized energy (35) can be written as an expansion

$$
\tilde{E} \simeq a_{0}+a_{1} x+a_{2} x^{2}+a_{3} x^{3} \quad(x \rightarrow \pm 0)
$$

in which the coefficients are

$$
a_{0}=p+\frac{q}{2}, \quad a_{1}=\frac{1}{2}, \quad a_{2}=-\frac{p+2 q}{16 p q}, \quad a_{3}=\left(\frac{p+2 q}{8 p q}\right)^{2} .
$$

Respectively, the strong-coupling limit for the energy can be represented as

$$
\tilde{E} \simeq b_{0} x^{2 / 5}+b_{1} x^{-2 / 5}+b_{2} x^{-6 / 5}+b_{3} x^{-2},
$$

where the coefficients are

$$
\begin{gathered}
b_{0}=\frac{5}{4}\left(p^{2} q\right)^{1 / 5}, \quad b_{1}=\frac{2 p^{2}+q^{2}}{4\left(p^{2} q\right)^{1 / 5}}, \\
b_{2}=-\frac{3 p^{4}-2 p^{2} q^{2}+2 q^{4}}{20\left(p^{2} q\right)^{3 / 5}}, \quad b_{3}=\frac{2 p^{6}-p^{4} q^{2}-2 p^{2} q^{4}+2 q^{6}}{20 p^{2} q} .
\end{gathered}
$$

These expansions are valid for arbitrary energy levels. The quantum numbers enter through notation (30). 


\section{Appendix B. Expansions for Attractive Interactions}

For atoms with attractive interactions, it is convenient to use the relation $x=-|x|$ and to express all quantities as functions of $|x|$. In expression (45) for the control function $u$, the coefficients are

$$
\begin{gathered}
u_{0}=1, \quad u_{1}=\frac{1}{2}, \quad u_{2}=\frac{1+3 \lambda}{8 \lambda}, \quad u_{3}=\frac{(1+2 \lambda)(3+10 \lambda)}{64 \lambda^{2}}, \\
u_{4}=\frac{2+20 \lambda+48 \lambda^{2}+35 \lambda^{3}}{128 \lambda^{3}}, \quad u_{5}=\frac{15+336 \lambda+1400 \lambda^{2}+2048 \lambda^{3}+1008 \lambda^{4}}{4096 \lambda^{4}}, \\
u_{6}=\frac{(1+\lambda)\left(35+221 \lambda+409 \lambda^{2}+231 \lambda^{3}\right)}{1024 \lambda^{4}} .
\end{gathered}
$$

Expansion (46) for the control function $v$ has the coefficients

$$
\begin{gathered}
v_{0}=1, \quad v_{1}=\frac{1}{2 \lambda}, \quad v_{2}=\frac{1+\lambda}{4 \lambda^{2}}, \quad v_{3}=\frac{7+20 \lambda+12 \lambda^{2}}{64 \lambda^{3}}, \\
v_{4}=\frac{5+32 \lambda+48 \lambda^{2}+20 \lambda^{3}}{128 \lambda^{4}}, \quad v_{5}=\frac{39+616 \lambda+1800 \lambda^{2}+1792 \lambda^{3}+560 \lambda^{4}}{4096 \lambda^{5}}, \\
v_{6}=\frac{35+192 \lambda+350 \lambda^{2}+256 \lambda^{3}+63 \lambda^{4}}{512 \lambda^{5}} .
\end{gathered}
$$

And in expansion (47) for the optimized energy, the coefficients are

$$
\begin{gathered}
c_{0}=\frac{2+\lambda}{2}, \quad c_{1}=-\frac{1}{2}, \quad c_{2}=-\frac{1+2 \lambda}{16 \lambda}, \quad c_{3}=-\frac{(1+2 \lambda)^{2}}{64 \lambda^{2}}, \\
c_{4}=-\frac{1+8 \lambda+16 \lambda^{2}+10 \lambda^{3}}{256 \lambda^{3}}, \quad c_{5}=-\frac{3+56 \lambda+200 \lambda^{2}+256 \lambda^{3}+112 \lambda^{4}}{4096 \lambda^{4}}, \\
c_{6}=-\frac{(1+\lambda)^{2}\left(5+22 \lambda+21 \lambda^{2}\right)}{1024 \lambda^{4}} .
\end{gathered}
$$

These expansions are written here for the ground-state level, which looses stability before other states with higher energies for $x \rightarrow x_{c}$ and $g \rightarrow g_{c}$, when attractive interactions become too strong. This is easy to understand keeping in mind that with increasing $|x|$ or $|g|$, that is, decreasing $x$ and $g$, all energy levels move down. Consequently, the lowest energy level will be the first to touch zero and then become complex, provoking the system instability. 


\section{Appendix C. Properties of Critical Lines}

The critical line $x_{c}(\lambda)$ is obtained as is explained in Sec. IV, from analysing the behaviour of the related self-similar factor approximants. On the line $x_{c}(\lambda)$, the ground-state energy becomes complex-valued. Generally, for a $k$-th order factor approximant, we get a $k$-th order critical line $x_{c}^{(k)}(\lambda)$ and, respectively, the effective-coupling critical line

$$
g_{c}^{(k)}(\lambda)=\frac{(2 \pi)^{3 / 2}}{2 \sqrt{\lambda}} x_{c}^{(k)}(\lambda)
$$

In the second order, we have

$$
x_{c}^{(2)}(\lambda)=-\frac{2 \lambda}{1+2 \lambda},
$$

which is the critical line (62). In the third order, we get

$$
x_{c}^{(3)}(\lambda)=-\frac{16 \lambda}{9+16 \lambda},
$$

which is very close to $x_{c}^{(2)}$. And for the fourth order, we find

$$
x_{c}^{(4)}(\lambda)=-\frac{8 \lambda(1+4 \lambda)}{\sqrt{2\left(19+36 \lambda+56 \lambda^{2}+64 \lambda^{3}\right)}-2+20 \lambda+32 \lambda^{2}} .
$$

The higher-order expressions $x_{c}^{(k)}(\lambda)$ become quickly so much cumbersome that the whole idea of deriving simple analytical expressions, allowing for an easy analysis, looses grounds. This is why, in the main text of the paper the simplest forms for the critical lines (62) and (63), corresponding to the second-order approximations, were used. In addition, the functions $g_{c}^{(k)}(\lambda)$ of all orders demonstrate rather similar behaviour. For instance, for the absolute values of the critical lines $g_{c}^{(k)}(\lambda)$, we obtain the following asymptotic behaviour in the limits of the small and large anisotropy parameter $\lambda$.

In the limit $\lambda \rightarrow 0$, related to a very elongated cigar-shaped trap, we have

$$
\begin{aligned}
& \left|g_{c}^{(2)}(\lambda)\right| \simeq 15.7496 \lambda^{1 / 2}-31.4992 \lambda^{3 / 2}+62.9984 \lambda^{5 / 2} \\
& \left|g_{c}^{(3)}(\lambda)\right| \simeq 13.9997 \lambda^{1 / 2}-24.8883 \lambda^{3 / 2}+44.2458 \lambda^{5 / 2} \\
& \left|g_{c}^{(4)}(\lambda)\right| \simeq 15.1278 \lambda^{1 / 2}-33.3560 \lambda^{3 / 2}+67.7766 \lambda^{5 / 2}
\end{aligned}
$$

In the opposite limit $\lambda \rightarrow \infty$, which refers to a very plane disk-shaped trap, we obtain

$$
\begin{aligned}
& \left|g_{c}^{(2)}(\lambda)\right| \simeq 7.8748 \lambda^{-1 / 2}-3.9374 \lambda^{-3 / 2}+1.9687 \lambda^{-5 / 2} \\
& \left|g_{c}^{(3)}(\lambda)\right| \simeq 7.8748 \lambda^{-1 / 2}-4.4296 \lambda^{-3 / 2}+2.4916 \lambda^{-5 / 2} \\
& \left|g_{c}^{(4)}(\lambda)\right| \simeq 7.8748 \lambda^{-1 / 2}-1.9687 \lambda^{-3 / 2}+1.7226 \lambda^{-5 / 2}
\end{aligned}
$$

Note that the limits

$$
\begin{gathered}
\lim _{\lambda \rightarrow \infty} x_{c}^{(k)}(\lambda)=-1, \\
\lim _{\lambda \rightarrow \infty} \sqrt{\lambda}\left|g_{c}^{(k)}(\lambda)\right|=\frac{(2 \pi)^{3 / 2}}{2}
\end{gathered}
$$


are the same in all orders.

Looking for the optimal trap shape at a fixed condensate density, that is, under a fixed frequency $\omega_{0}$, we have to study the behaviour of the effective coupling

$$
\alpha_{0}^{(k)}(\lambda)=\frac{\lambda^{1 / 6}}{4 \pi}\left|g_{c}^{(k)}(\lambda)\right|=\frac{\sqrt{2 \pi}}{4 \lambda^{1 / 3}}\left|x_{c}^{(k)}(\lambda)\right|
$$

The maximum of this quantity defines the critical number (58). For all orders $k$, the maximum of $\alpha_{0}^{(k)}(\lambda)$ happens at $\lambda \approx 1$. Thus, $\lambda^{(2)}=1, \lambda^{(3)}=1.1$, and $\lambda^{(4)}=0.9$. This means that, under a fixed condensate density, the spherical trap is optimal. 


\section{REFERENCES}

[1] A.S. Parkins and D.F. Walls, Phys. Rep. 303, 1 (1998).

[2] P.W. Courteille, V.S. Bagnato, and V.I. Yukalov, Laser Phys. 11, 659 (2001).

[3] L. Pitaevskii, and S. Stringari, Bose-Einstein Condensation (Clarendon, Oxford, 2003).

[4] J.O. Andersen, Rev. Mod. Phys. 76, 599 (2004).

[5] K. Bongs and K. Sengstock, Rep. Prog. Phys. 67, 907 (2004).

[6] R.A. Duine and H.T.C. Stoof, Phys. Rep. 396, 115 (2004).

[7] V.I. Yukalov, Laser Phys. Lett. 1, 435 (2004).

[8] N.N. Bogolubov, Lectures on Quantum Statistics (Gordon and Breach, New York, 1967), Vol. 1.

[9] N.N. Bogolubov, Lectures on Quantum Statistics (Gordon and Breach, New York, 1970), Vol. 2.

[10] D. ter Haar, Lectures on Selected Topics in Statistical Mechanics (Pergamon, Oxford, 1977).

[11] C.C. Bradley, C.A. Sackett, J.J. Tollett, and R.G. Hulet, Phys. Rev. Lett. 75, 1687 (1995)

[12] C.C. Bradley, C.A. Sackett, and R.G. Hulet, Phys. Rev. Lett. 78, 985 (1997).

[13] J.M. Gerton, D. Strekalov, I. Prodan, and R.G. Hulet, Nature 408, 692 (2000).

[14] J.L. Roberts, N.R. Claussen, S.L. Cornish, E.A. Donley, E.A. Cornell, and C.E. Wieman, Phys. Rev. Lett. 86, 4211 (2001).

[15] E.A. Donley, N.R. Claussen, S.L. Cornish, J.L. Roberts, E.A. Cornell, and C.E. Wieman, Nature 412, 295 (2001).

[16] N.R. Claussen, E.A. Donley, S.T. Thompson, and C.E. Wieman, Phys. Rev. Lett. 89, 010401 (2002).

[17] P.A. Ruprecht, M.J. Holland, K. Burnett, and M. Edwards, Phys. Rev. A 51, 4704 (1995).

[18] R.J. Dodd, M. Edwards, C.J. Williams, C.W. Clark, M.J. Holland, P.A. Ruprecht, and K. Burnett, Phys. Rev. A 54, 661 (1996).

[19] M. Houbiers and H.T.C. Stoof, Phys. Rev. A 54, 5055 (1996).

[20] V.I. Yukalov, E.P. Yukalova, and V.S. Bagnato, Phys. Rev. A 56, 4845 (1997).

[21] N. Akhmediev, M.P. Das, and A.V. Vagov, Int. J. Mod. Phys. B 13, 625 (1999).

[22] C. Huepe, S. Metens, G. Dowel, P. Borckmans, and M.E. Brachet, Phys. Rev. Lett. 82, 1616 (1999).

[23] M. Wadati and T. Tsurimi, J. Phys. Soc. Jpn. 68, 3840 (1999).

[24] L. Bergé, T.J. Alexander, and Y.S. Kivshar, Phys. Rev. A 62, 023607 (2000).

[25] V.M. Pérez-Garcia, H. Michinel, and H. Herrero, Phys. Rev. A 57, 3837 (1998).

[26] A. Gammal, T. Frederico, and L. Tomio, Phys. Rev. A 64, 055602 (2001).

[27] A. Gammal, L. Tomio, and T. Frederico, Phys. Rev. A 66, 043619 (2002).

[28] V.I. Yukalov, Moscow Univ. Phys. Bull. 31, 10 (1976).

[29] V.I. Yukalov and E.P. Yukalova, Ann. Phys. (N.Y.) 277, 219 (1999).

[30] V.I. Yukalov and E.P. Yukalova, Chaos Solitons Fractals 14, 839 (2002).

[31] B. Kastening, Phys. Rev. A 68, 061601 (2003).

[32] B. Kastening, Laser Phys. 14, 586 (2004).

[33] B. Kastening, Phys. Rev. A 69, 043613 (2004).

[34] J.L. Kneur, A. Neveu, and M.B. Pinto, Phys. Rev. A 69, 053624 (2004). 
[35] B. Kastening, Phys. Rev. A 70, 043621 (2004).

[36] J.L. Kneur and M.B. Pinto, Phys. Rev. A 71, 033613 (2005).

[37] P. Arnold and G. Moore, Phys. Rev. Lett. 87, 120401 (2001).

[38] P. Arnold and G. Moore, Phys. Rev. E 64, 066113 (2001).

[39] V.A. Kashurnikov, N. Prokofiev, and B. Svistunov, Phys. Rev. Lett. 87, 120402 (2001).

[40] N. Prokofiev and B. Svistunov, Phys. Rev. Lett. 87, 160601 (2001).

[41] K. Nho and D.P. Landau, Phys. Rev. A 70, 053614 (2004).

[42] V.I. Yukalov, Phys. Rev. A 42, 3324 (1990).

[43] V.I. Yukalov, Physica A 167, 833 (1990).

[44] V.I. Yukalov, J. Math. Phys. 32, 1235 (1991).

[45] V.I. Yukalov, J. Math. Phys. 33, 3994 (1992).

[46] V.I. Yukalov and E.P. Yukalova, Physica A 225, 336 (1996).

[47] V.I. Yukalov, S. Gluzman, and D. Sornette, Physica A 328, 409 (2003).

[48] S. Gluzman, V.I. Yukalov, and D. Sornette, Phys. Rev. E 67, 026109 (2003).

[49] V.I. Yukalov and S. Gluzman, Int. J. Mod. Phys. B 18, 3027 (2004).

[50] C.C. Bradley, C.A. Sackett, and R.G. Hulet, Phys. Rev. A 55, 3951 (1997). 